\title{
QUALITY VERSUS QUANTITY
}

Q uality Adjusted Life Years - QALYs - have been in the news lately because they offer a rational approach to the allocation of resources in health care. Spending the limited health care dollars on the cheapest QALYs will maximise the health gains obtained from the health care budget. Therefore reviewing spending in terms of its cost per QALY has a lot of appeal to those now confronting health care rationing.

Quality Adjusted Life Years are life years weighted to reflect their quality. QALYs were developed as a measure of benefit for use in economic evaluation. Cost benefit analysis, historically the first type of economic evaluation, requires that both costs and benefits are measured in money terms. That presents obvious difficulties in its application to health care where the benefits are the extension of life, the relief of pain and the reduction of disability. In health care, cost effectiveness analysis has been more widely applied than cost benefit analysis. In cost effectiveness analysis, the benefits of health care are measured in their naturally occurring "units" - most generally number of life years saved.

The problem with number of life years gained is that they do not capture all the benefits of health care, such as improved mobility after hip replacement. Nor do they distinguish between lives of different quality such as a normally functioning child compared to a child with blindness or intellectual handicap - both of which could be the outcome of neonatal intensive care. Life years gained fail to encompass all the benefits of health care interventions, where quality of life is the important objective, or to make explicit tradeoffs between quality and quantity of life.

QALYs were developed in an attempt to overcome these problems in cost effectiveness analysis. They measure quality of life on the same scale as quantity of life, so multiple objectives, such as prolonging life and improving its quality, can be combined in a single measure. The weight applied to life years to adjust them for quality is also referred to as a utility; hence the term cost utility analysis is often used to describe the form of economic evaluation in which QALYs are used to measure benefits ${ }^{1}$.

The difference that QALYs might make is illustrated in Figure 5 . The number of life years gained from treatment $\mathrm{X}$ is shown on the $\mathrm{X}$-axis; in this example it is 15. If quality is also taken into account, as shown on the Y-axis, then the relevant measure is the area under the curve; in this example it is 9 QALYs.

\section{MEASUREMENT PROPERTIES OF QALYS}

The need to combine quantity and quality in one scale gives rise to some special measurement properties required for QALYs. First, quality must be measured by a weight between 0 representing no life years, and 1 representing fully functional life years. So the measurement of quality of life must be anchored to death -0 and full health -1 . Note this does not preclude health states worse than death.

Second, the scale must have interval properties. That means a movement from .3 to .5 must be the same as a movement from .7 to .9 and both must be twice the value of a movement from .9 to 1.0. This ensures that $.3 \times 5$ life years is worth the same as $.5 \times 3$ life years.

There are several hundred scales which attempt to measure health related quality of life or health status. Examples include the Sickness Impact Profile, the Index of Activities of Daily Living ${ }^{3}$ and the Spitzer QL Index. But very few of these have the measurement properties such as interval scaling anchored to death and full health required for QALYS. Many of the scales are objective in that they try to measure what individuals can do, rather than their feelings. The authors of the Sickness Impact Profile, for example, state quite clearly that this instrument is measuring the behavioural impact of sickness on individuals and not how those individuals feel about their health state. In contrast, the purpose of QALYs is to measure how individuals feel about health states, or the strength of their preferences for health outcomes. QALYs are inherently subjective.

\section{DO QALYS MEASURE QUALITY OF LIFE?}

Quality of life, as the phrase is generally used, includes health but goes beyond health status to encompass economic and material circumstances, personal relationships, the physical environment and more. Clearly QALYs do not purport to embrace all of this. But the term quality adjusted and the use of quality of life as a synonym for it, has led to some confusion.

Some researchers have suggested that an alternative phrase would reduce this. Mehrez and Gafni have coined the phrase healthy year equivalent, while others use the phrase disability-free life expectancy.

There are two approaches to measuring QALYs: use one of the measures of health related quality of life that has the appropriate scaling characteristics, or measure health state preferences directly.

Three measures of health related quality of life are appropriate: the Quality of Well Being Index of Kaplan and Bush, the Rosser scale ${ }^{6}$, and the Multi-Attribute Utility model of Torrance1. The Rosser scale, as modified by Williams and his colleagues at York, has been widely used in cost utility work in the UK to the extent that QALYs and the Rosser-York scale have become synonymous. Using an existing measure requires the health outcomes under consideration to be described in terms of that measure. But a general measure may be insensitive to some aspect of quality of life that is specific to a certain condition. For example, patients with cancer often report feeling socially isolated and treated as though cancer were contagious. None of the instruments cited is sensitive to this.

The alternative approach is to measure directly preferences for specific health outcomes. There are a number of measurement techniques, the most common being the standard gamble and the time trade-off. Under the former, individuals are asked to choose between a gamble on good health versus death and a life spent in a chronic ill-health state. The time trade-off requires individuals to trade off years in a chronic ill-health state for a shorter time in good health. The direct measurement of preferences is associated most strongly with the work of Torrance and his colleagues at McMaster University in Canada. 


\section{Quality versus Quantity}

\section{Continued from page 115}

This approach can involve quite extensive survey work to elicit preferences from patients and/or a general sample of the community. A short-cut is to use professional judgment, as exemplified in the work of Weinstein from Harvard?

Whichever approach is taken to measuring QALYs, cost utility analysis requires far more extensive data on the outcomes of health care and their duration than exist generally at present.

\section{CRITICISMS OF QALYS}

It is important to distinguish criticisms of the measurement methods themselves from criticisms of the concept of QALYs per se. The Rosser-York scale has been extensively criticised on the basis of the measurement technique and the smallness of the sample used to derive the initial weights, and the apparent lack of sensitivity in those weights to changes in health state. More recent empirical work from York has thrown doubt on the validity of the initial weights.

Of all the approaches, it is not clear which should be accepted as the "gold standard", yet different measurement techniques give different answers. This has led some commentators to question why anyone bothers. The answer to that is simple: there are significantly different values attached to different outcomes, whatever technique is used. It is obvious that health related quality of life does matter.

What of the concept of the QALY itself? QALYs have been criticised as being biased against the elderly, as they have far fewer QALYs to gain than the young. It is true that a life saved at age 60 will result in fewer life years gained than a life saved at age 30 - if both live their normal lifespan. It is important to remember that

\section{FIGURE 5}

\section{QALYS}

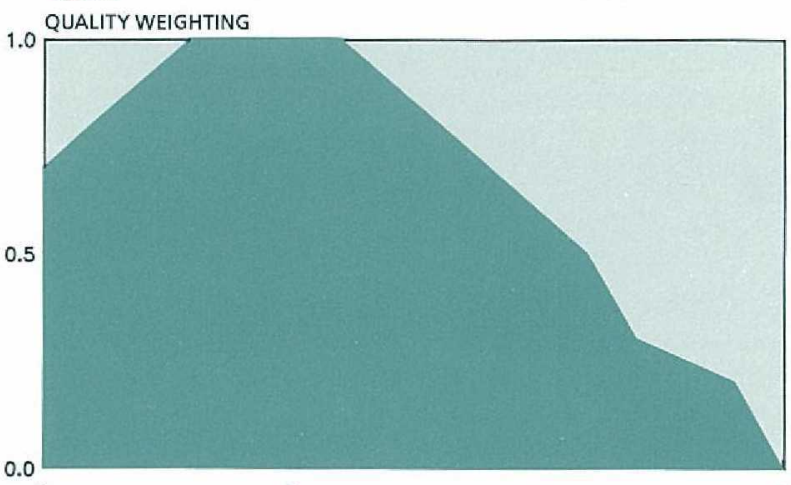

15 it is life years gained that are considered here, not total future life years. Quality adjusting those life years takes into account the relief of chronic non-fatal disease which is more likely to be experienced by the elderly.

Comparisons on the basis of cost per QALY have shown that hip replacements and chiropody for over 75-yearolds are much more efficient than transplants or heart surgery.

QALYs are egalitarian in that a QALY gained is a QALY gained irrespective of who receives it. This egalitarian distribution rule may not reflect social attitudes of what is the desired distribution. Gains for the elderly may not be valued as highly as gains for the young; gains for the very young may not be valued as highly as gains for the adult with family responsibilities.

The distribution of QALY gains is not the only equity concern of health care systems. Most are concerned with providing access to health care services, and QALYs simply do not capture those other equity objectives. QALYs are a measure of health care efficiency and do not account for equity.

QALYs may not be the only output of health care. The provision of information itself may be important; how else do the "worried" determine whether they are sick or well. Other aspects of health care provision that can be thought of more as process than outcome are being treated as autonomous, with dignity and being cared for; and these are not captured by QALYs.

QALYs are an important advance in conceptualising and measuring the output of the health system. But they cannot yet be regarded as having progressed beyond the experimental stage and further developmental work is required. Far more extensive data collection is required on the outcomes, including their durations, of health care.

While QALYs may never encompass all the important and legitimate objectives of health services, notably those that pertain to equity and to care rather than cure, they do require a more explicit base for decision making. Economic evaluation is an aid to decision making. It is a major advance on resource allocation by shroud waving.

Jane Hall, Centre for Health Economics Research and Evaluation.

1. Drummond MF, Stoddart GL, Torrance GW. Methods for the Economic Evaluation of Health Care Programs. Oxford Medical Publications. 1987. 2. Bergner M, Bobbitt RA, Carter NB and Gilson BS. The Sickness Impact Profile: development and final revision. Medical Care $1981 ; 19: 787-805$

3. Katz S, Ford AB, Moskowitz RW et al. Studies of illness in the aged: the index of ADL, a standardised measure of biological and pyschosocial function. $J$ Am Med Assoc 1963; 185:94.

4. Spitzer WO, Dobson AJ, Hall J et al. Measuring the quality of life of cancer patients: a concise index for use by physicians. J of Chronic cancer patients: a concis

5 . Kaplan RM, Bush JW. Health related quality of life measurement for evaluation research and policy analysis. Health Psychology 1982; evaluation 61.80 .

6. Gudex $\mathrm{C}$ and Kind P. The QALY toolkit. Centre for Health Economics Discussion Paper 38

7. Hatziandreu EI, Koplan JP, Weinstein MC et al. A cost effectiveness analysis of exercise as a health promotion activity. Am J of Pub Health $1988 ; 78(11): 1417-1421$

8. Mooney GH. QALYs: are they enough? A health economist's perspective. J of Medical Ethics 1989; 15:148-152. 